\title{
Variations in the use of coronary angiography in three cities in the Trent Region
}

Division of Cardiovascular Medicine, Department of Medicine, University Hospital, Queen's Medical Centre, Nottingham D Gray

J R Hampton

Correspondence to: Dr D Gray, Division of Cardiovascular Medicine, Department of Medicine, University Hospital, Queen's Medical Centre Nottingham NG7 2UH. Accepted for publication 1 November 1993

\begin{abstract}
Objective-To establish the characteristics of patients referred for coronary angiography and the outcome of investigation.

Design-Prospective study.

Setting-Three regional referral centres at Sheffield, Leicester, and Nottingham.

Patients-All patients referred for investigation from 1 July 1988 to 30 June 1989.

Interventions-Coronary angiography for suspected ischaemic heart disease.

Main outcome measures-Site and extent of coronary artery disease at coronary angiography and subsequent intervention.

Results-There was a range of clinical activity with a trend towards symptomatic control in Nottingham where patients tended to have more severe angina of long duration and extensive drug treatment. Important coronary lesions were often found and most patients needed coronary artery bypass surgery or angioplasty. In Leicester and Sheffield, where angiography was used prognostically, patients tended to have mild angina of shorter duration and less extensive medical treatment; significant coronary disease was often found but fewer patients were recommended for further intervention.

Conclusions-Referral for coronary angiography seems to reflect philosophical differences among the referring physicians. Referring patients late in the disease process ensures that most have a subsequent intervention but the benefits of revascularisation may be denied to those with mild symptoms but extensive coronary disease.
\end{abstract}

(Br Heart f 1994;71:474-478)

The use of coronary angiography in the management of ischaemic heart disease varies widely-for example, the United States performs about three times the number of procedures carried out in the United Kingdom ${ }^{1}$ and Canada more than twice, ${ }^{2}$ but even within the United States of America the number of procedures carried out in each state differs. $^{3}$
A target rate of 300 coronary artery bypass operations per million of the population has been proposed ${ }^{4}$ but without recommendation on whom should be investigated. An expert panel of doctors involved in the clinical care and investigation of patients with ischaemic heart disease met to evaluate hypothetical indications (or clinical scenarios) for coronary angiography; the notes of patients who had recently had angiography were reviewed and the panel's evaluations of the hypothetical indications were applied to the actual patients to get a measure of appropriateness for each clinical case. ${ }^{5}$ The degree of appropriateness of the procedure differed significantly between the three designated cardiac investigation centres in the Trent Region from 37\% to $63 \%$, a highly significant difference.

The centre with evaluations least in agreement with the consensus panel was Sheffield, the centre with evaluations nearest to those of the panel was Nottingham, and the third centre, Leicester, was in between.

Being considered appropriate does not necessarily mean that the decision to investigate was correct, nor does an inappropriate evaluation mean that the decision was wrong. A different panel might have produced slightly different evaluations, and the limited data from clinical trials on which doctors currently base their clinical decisions involved selective populations that are probably not representative of the wide range of patients who appear in hospital clinics.

From this study we suspected that the process to select patients with suspected myocardial ischaemia for cardiac catherisation varied in the different referral centres within the Trent Region, at Sheffield, and Leicester where cardiac surgery is available, and Nottingham where surgery is not available. A more comprehensive study of patients undergoing invasive investigation in our region was undertaken firstly to establish whether different sorts of patients were being referred for coronary angiography, and secondly to ascertain the outcome of the investigation.

\section{Patients and methods}

METHOD

Data were collected prospectively from all patients undergoing coronary angiography in the three regional centres at Sheffield, 
Leicester, and Nottingham by research assistants participating in the British Cardiac Society Randomised Intervention Treatment of Angina (RITA) study. Data from RITA were supplemented by detailed information from the patient record at or shortly after the catheterisation procedure. Notes of all patients undergoing coronary angiography between 1 July 1988 and 30 June 1989 were reviewed.

Coronary angiograms were reported at the centre that performed the procedure, and the following criteria were applied: "There must be significant stenosis in one, two, or three coronary arteries or major branches. A stenosis is "significant" if there is at least a $70 \%$ reduction in diameter in one angiographic view, or $50 \%$ reduction in two views; the stenosed vessel must be judged to supply $20 \%$ or more of the left ventricular muscle".

Patients were identified according to their postcode as living within the city boundary of Sheffield, Leicester, or Nottingham; patients who were not city residents were excluded from the study, because our previous experience suggested that proximity to a centre itself might have a considerable influence on the use of a procedure. ${ }^{5}$ The characteristics of patients (resident within the city) were similar to those excluded (resident outside the city).

All patients were followed up for at least one year after the cardiac catheterisation. In some cases data were incomplete but there was a core of information available for all patients for analysis.

Statistical analysis was by $\chi^{2}$ test; only conventional significance levels $\mathrm{p}<0.05$ are shown.

The British Heart Foundation Statistics Unit at Nottingham University supervised all aspects of data handling.

\section{Results}

During the period of data collection 2096 cardiac catherisation procedures that included coronary angiography were performed in the three regional referral centres for cardiac catheterisation. A total of 699 procedures were performed at Sheffield, 1001 at Leicester, and 396 at Nottingham. Of these, 636 were excluded from the analysis because the primary reason for investigation was not suspected ischaemic heart disease-432 catherisations were arranged primarily to investigate valvar disorders; 156 to assess diverse conditions including congenital heart disease, cardiomyopathy, and assessment for cardiac transplantation; and 48 patients had been referred from outside the Trent Region.

Cardiac catheterisation was carried out on 1460 patients to investigate symptoms suggestive of ischaemic heart disease. The $\mathbf{7 8 5}$ patients referred from outside the cities were excluded from further analysis. The remaining 675 patients were investigated within their own home city, with 150 catherised in Sheffield, 371 in Leicester, and 154 in Nottingham; these patients form the basis of this study.

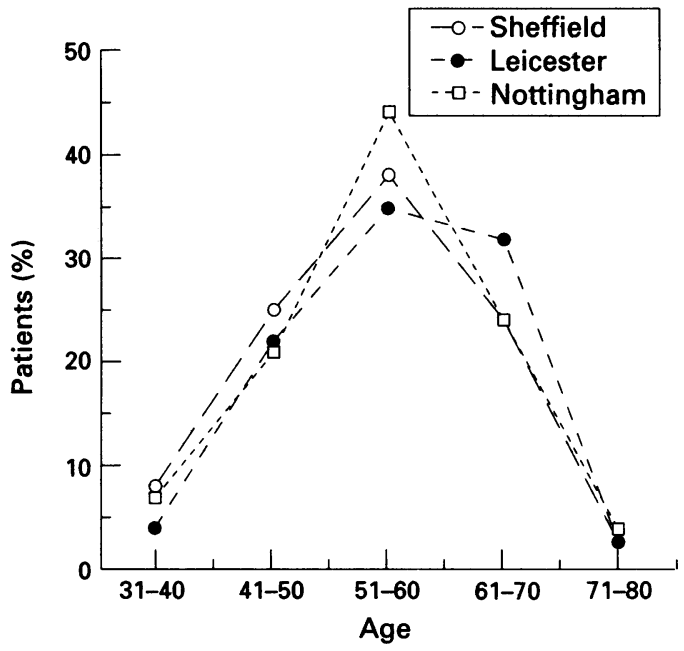

Figure 1 Age distribution of patients.

\section{PATIENT DEMOGRAPHY}

Figure 1 shows that the age distribution of patients investigated in each centre was similar, with few patients under 40 and very few over 80 . The mean age was 54 years in Sheffield, 56 in Leicester, and 56 in Nottingham. The ratio of men to women was 1.3:1 in Sheffield, 0.75:1 in Leicester and $1 \cdot 5: 1$ in Nottingham.

Figure 2 shows the employment of patients. Overall, $34 \%$ of patients were in regular employment, $15 \%$ were off work due to ill health, and $40 \%$ were retired, more than one third of them on health grounds. Patients from Leicester were more likely to be in work than patients from elsewhere. Overall, $5 \%$ of patients held either a heavy goods vehicle, public service vehicle, or pilots' licence at the time of catherisation.

\section{DURATION OF SYMPTOMS}

Figure 3 shows that few patients had had symptoms of chest pain for less than six months and most patients had had symptoms for over 12 months. Patients from Nottingham tended to have had symptoms for longer than those from the surgical centres.



Figure 2 Employment of patients. 
Figure 3 Duration of symptoms.

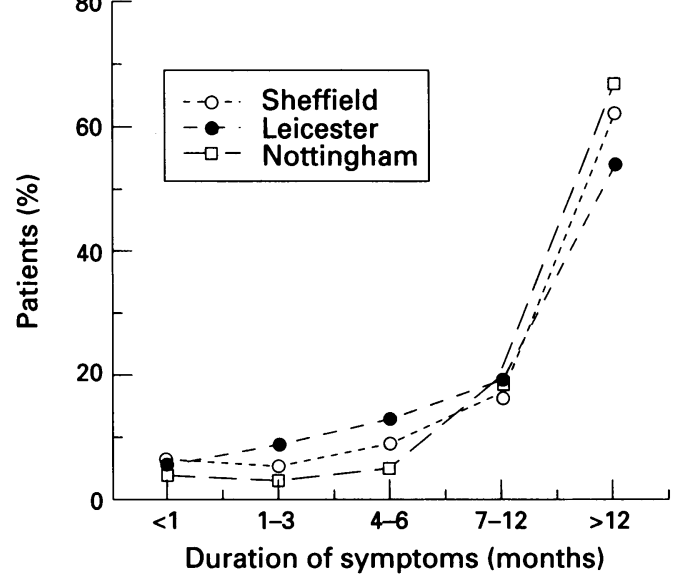

INDICATION FOR CORONARY ANGIOGRAPHY The most often documented reason for carrying out angiography was to investigate chest pain thought to be due to chronic stable angina, with only a few investigations soon after an acute myocardial infarction.

Most patients had persistent pain despite medication when presenting for angiography. Twelve per cent of patients from Leicester were pain free at the time of cardiac catherisation, compared with $2 \%$ in Sheffield and $2 \%$ in Nottingham.

\section{EVENTS BEFORE CATHETERISATION}

A history of a previous myocardial infarction was a common finding in patients from all areas, with $54 \%$ of patients from Nottingham having had a myocardial infarction before catheterisation compared with $46 \%$ from Leicester and $41 \%$ from Sheffield.

Seventy four (11\%) patients had had an episode of unstable angina in the six months before coronary angiography. About half of these had had a cardiac catheterisation when in hospital with unstable symptoms. Sheffield doctors were much more likely to arrange a cardiac catheterisation when their patients had been admitted to hospital than were those at the other centres- $21 \%$ of Sheffield patients had a cardiac catheterisation, $7 \%$ of those from Leicester, and 10\% from Nottingham.

Coronary artery bypass surgery had pre-

Figure 4 Severity of symptoms. viously been carried out in $8 \%$ of patients from Sheffield, $10 \%$ from Leicester, and 5\% from Nottingham. Previous percutaneous transluminary coronary angioplasty had been performed on $6 \%$ of patients from Sheffield, $5 \%$ from Leicester, and 1\% from Nottingham.

Figure 4 shows the severity of symptoms. According to the New York Heart Association (NYHA) criteria, more Nottingham patients had symptoms of severe angina than patients from Sheffield or Leicester. Among the Leicester patients, most patients had grade II symptoms and only $12 \%$ had grade IV angina. Among the patients investigated at Sheffield, grade II and grade III symptoms were the most frequent, and $22 \%$ had grade IV symptoms. In contrast, most of the patients from Nottingham had grade III and grade IV symptoms. These findings were highly significant $\mathrm{p}<0.001$.

\section{MEDICATION BEFORE CATHETERISATION}

Table 1 shows how often the three main classes of drugs were prescribed for patients from each of the three centres. Prescribing preferences were evident in the three centres. The most often prescribed drug taken by almost three quarters of all patients was a $\beta$ antagonist, then a long acting nitrate, and a calcium antagonist. $\beta$ Antagonists were used preferentially by patients from Leicester and Nottingham but patients from Sheffield were more likely to be taking a calcium antagonist. As a second drug, Leicester patients commonly took a long acting nitrate, whereas Nottingham patients used a calcium antagonist. These differences in prescribing habit were highly significant $p<0 \cdot 02$.

Overall, $6 \%$ of patients were on no regular medication at all at the time of the cardiac catheterisation, $21 \%$ of patients were taking at least one drug to control symptoms, $27 \%$

Table 1 Proportions of patients taking specific medication at the time of cardiac catheterisation

\begin{tabular}{llll}
\hline & Sheffield & Leicester & Nottingham \\
\hline$\beta$ antagonist & 59 & 73 & 81 \\
Calcium antagonist & 64 & 53 & 71 \\
Long acting nitrate & 64 & 47 & 80
\end{tabular}
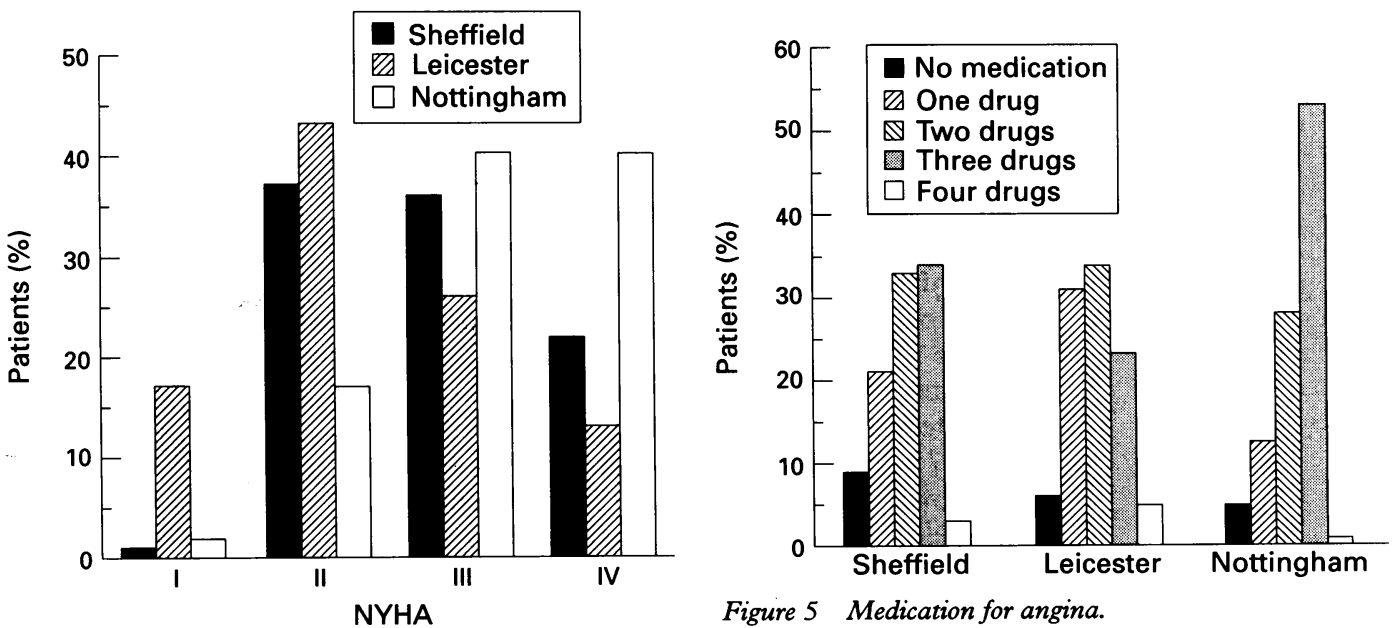

Figure 5 Medication for angina. 
at least two, and $36 \%$ three types of drugs (fig 5). Also, a few were taking a diuretic as well as antianginal agents. Combinations of two or three drugs were more likely to be prescribed for patients from Nottingham than for patients from the other cities $(p<0.01)$.

INVESTIGATIONS BEFORE CATHETERISATION

Before catheterisation, about two thirds of all patients had an exercise test; $30 \%$ of patients from Sheffield, $69 \%$ from Leicester, and $65 \%$ from Nottingham. Where tests were carried out, these were described as positive for myocardial ischaemia in $74 \%$ performed in Sheffield, $68 \%$ of those in Leicester, and $75 \%$ in Nottingham.

Of Nottingham patients $43 \%$ had tests considered positive at a low level-that is, showing evidence of myocardial ischaemia in stage 1 or earlier with the Bruce protocol or its equivalent on the Sheffield protocol, compared with $38 \%$ from Sheffield and $32 \%$ from Leicester.

Exercise thallium, ${ }^{99} \mathrm{Tc}$ methoxy-isobutyl isonitryl (MIBI), and multigated acquisition (MUGA) scans were rarely requested in any centre.

\section{FINDINGS AT ANGIOGRAPHY}

Fourteen per cent of all coronary angiograms did not show coronary artery disease severe enough to require any intervention (table 2). More patients from Sheffield were thought to have a normal angiogram than patients from elsewhere. Significant coronary artery disease affecting all three major coronary vessels was found in one third of patients from Nottingham, a quarter from Sheffield, and one in 10 from Leicester; significant left main stem disease was found more often in Leicester patients than in patients at the other centres, and lesions of the proximal left anterior descending artery were found in almost half of all patients.

Some degree of left ventricular impairment was found in almost half of all patients, but severe dysfunction was found infrequently.

There was no significant difference among the three centres in their ability to identify patients with coronary artery disease affecting the left main stem. The surgical centres were no more likely to find coronary disease than the non-surgical centre.

\section{MANAGEMENT}

The management options available at the time of this series of cardiac catheterisations were elective coronary artery bypass surgery, elective percutaneous transluminal coronary

Table 2 Findings at cardiac catheterisation

\begin{tabular}{lcrl}
\hline & Sheffield & Leicester & Nottingham \\
\hline Normal angiogram (\%) & $27(18)$ & $45(12)$ & $22(14)$ \\
Left main stem lesion (\%) & $13(9)$ & $40(12)$ & $11(7)$ \\
Proximal LAD lesion (\%) & $60(40)$ & $171(46)$ & $76(49)$ \\
Three vessel disease (\%) & $42(28)$ & $42(11)$ & $52(33)$ \\
Left ventricular dysfunction (\%): & & \\
Severe & 2 & 7 & 8 \\
Mod or mild & 41 & 44 & 22 \\
Normal & 57 & 48 & 69 \\
\hline
\end{tabular}

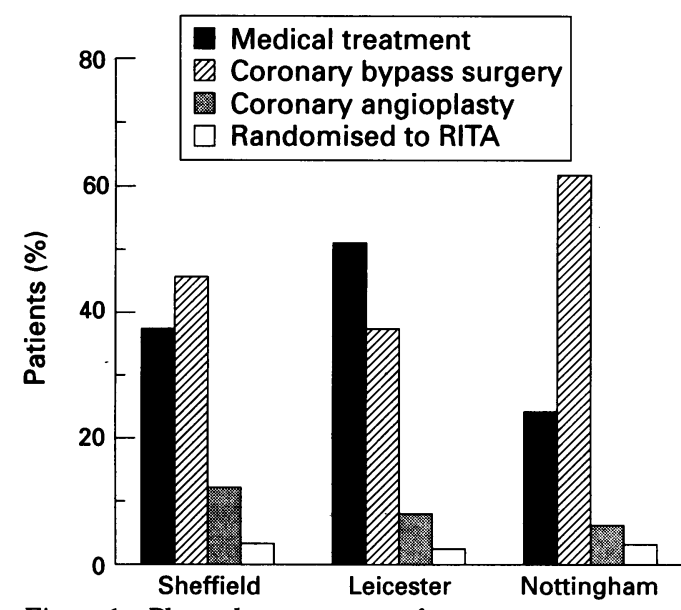

Figure 6 Planned management after coronary angiography.

angioplasty, randomisation to either surgery or angioplasty (as part of the RITA study), or continued medical treatment. Figure 6 shows that about one third of the patients from Sheffield were recommended to continue medical treatment; as were half of patients from Leicester and a quarter from Nottingham. Surgery or angioplasty was proposed in $60 \%$ of patients from Sheffield, $47 \%$ from Leicester, and $71 \%$ of patients from Nottingham $(p>0.001)$.

\section{Discussion}

A good history, physical examination, and non-invasive tests yield information that is of diagnostic and prognostic value, ${ }^{6}$ but only coronary angiography can define suitability for coronary revascularisation. Once the coronary anatomy is known, decision making may become easier, despite slightly conflicting results from the main trials. ${ }^{7-9}$ It is generally accepted that intervention is needed when drugs fail to control pain or to prolong survival in patients with more than $50 \%$ luminal narrowing of the left main stem, or with three vessel disease with impaired left ventricular function and significant narrowing of the proximal left anterior descending artery.

The indications for coronary angiography, however, are still unsettled, ${ }^{10}$ but in chronic stable angina the need for coronary angiography falls into three categories. Firstly, it may be performed to confirm clinically suspected coronary artery disease (as a prelude to bypass surgery), secondly to stratify risk for prognostic purposes, and thirdly to obtain an accurate diagnosis when other investigations have failed. Who should be considered for an angiogram probably depends on the philosophy held by the clinician. Those who seek to control symptoms may persist with escalating doses of up to three drugs until symptoms cannot be controlled with medication; those who are concerned about minimising risk can justify angiography at a much earlier stage to avoid missing patients with left main stem disease or extensive coronary disease; and all will see patients with an indeterminate diagnosis despite extensive tests for non-cardiac causes. 
This spectrum of clinical activity in relation to invasive investigation is seen in our survey, with a tendency towards symptomatic control in Nottingham and early angiographic prognosis in Leicester and Sheffield.

The patients from Nottingham had the most advanced ischaemic heart disease at the time of angiography. They tended to have had symptoms for longer than those from other centres, most had angina graded moderately severe or severe on NYHA criteria, they were more likely to have had a myocardial infarction at some time, they required extensive drug treatment to limit symptoms, and they often had early positive stress tests. Significant coronary lesions were commonly found at cardiac catheterisation and most were accepted for either coronary artery bypass surgery or angioplasty.

Leicester patients seemed to have milder degrees of coronary disease, perhaps at an earlier stage in its natural history as coronary disease was less extensive. For most patients symptoms were of shorter duration, angina was less severe, and more patients had no pain when admitted for cardiac catheterisation, fewer patients had two or three drugs to control symptoms, patients were more likely to be employed and performance on treadmill tests was better than in patients from the other cities. Investigation of these patients with milder symptoms did not increase the occurrence of a normal coronary angiogramLeicester had a low rate compared with other centres. Significant coronary lesions were as likely to be found, but only half were recommended for further intervention-it is not clear whether distal vasculature may have been considered unsuitable for coronary artery bypass grafting or percutaneous transluminary coronary angioplasty or whether the patients simply had milder symptoms.

Sheffield patients seem to have had milder disease than Nottingham but more severe disease than Leicester. Non-invasive investigation was used less often to assess patients with chronic stable angina, and this may have been responsible for the increased proportion of normal coronary arteries seen.

The Leicester approach of carrying out an angiogram at an early stage in the disease process not only established a firm diagnosis sooner than the other centres but also was more successful in identifying patients with significant disease of the left main stem than the other centres. It is possible that the relative lack of patients with left main stem disease in Sheffield and Nottingham occurred because these patients had died without investigation.

In Nottingham, angiography seems to be performed late in the disease process and referral for angiography is largely symptom dependent; although this ensures that most patients proceed to some form of revascularisation, it is not entirely satisfactory because some patients with relatively mild symptoms will have advanced coronary disease (such as left main stem or severe three vessel disease) as a result of which they may be denied access to surgery that might prolong life.

The initial impression that there were differences in the use of coronary angiography in the three cities in the Trent Region seems to be upheld, with angiography being used by some as a diagnostic test and by others as a prognostic indicator. Although both approaches are valid (seen in this study as the ability to detect coronary artery disease), reliance on symptoms can be rather limiting-they do not correlate closely with the extent of coronary disease, ${ }^{7}$ and the recognised benefits of bypass surgery with respect to mortality may be denied to patients with severe coronary disease whose symptoms happen to be mild.

Coronary angiography was readily available in all three centres and we think it likely that the variation in practice between the three centres that we have described was due more to the philosophy of the physician than to availability of resources. We cannot discount the possibility that the availability of surgery at Sheffield and Leicester might have influenced the behaviour of physicians, especially during an admision to hospital with unstable angina or where there is diagnostic uncertainty.

Our study does not help define ideal practice but it does point to the need for purchasers to have definite concepts of what they regard as acceptable indications for coronary angiography. If the purpose of health care is to improve outcome, ${ }^{11}$ not just to control symptoms, then the Leicester approach has much to commend it, but the only way that the best management can be determined satisfactorily is by a clinical trial.

We are grateful to the physicians and surgeons of the Trent Region who were either members of the appropriateness panel or who kindly allowed access to case notes.

1 National Centre for Health Statistics. National hospital discharge survey: annual summary, United States, 1987. Vital and health statistics. Series 13 no 99 . Washington: Government Printing Office 1989. (DHHS Publ no (PHS) 89-1760)

2 Naylor CD, Baigrie RS, Goldman BS, Basinski A. Assessment of priority for coronary revascularisation procedures. Lancet 1990;i:1070-3.

3 Chassin MR, Kosecoff J, Park RE, Fink AR, Rauchman $\mathrm{S}$, Keesey J, et al. Variations in the use of medical and surgical services by the Medicare population. Engl $f$ Med 1986;314:285-90.

4 UK Consensus Development Conference. Coronary artery bypass surgery: a consensus. Lancet 1984;ii: 1269. artery bypass surgery: a consensus. Lancet 1984;ii: 1269.
Gray D, Hampton JR, Bernstein SJ, Kosecoff J, Brook RH. Audit of coronary angiography and bypass surgery.
RH D RH. Audit of coronary angiog
Lancet 1990;335:1317-20.

Lancet 1990;335:1317-20.
Ambrose JA. Unsettled indications for coronary angiography. $尹$ Am Coll Cardiol 1984;3:1575-80.

7 Bruscke AVG, Proudfit WL, Sones FM. Progress study of 590 consecutive nonsurgical cases of coronary disease followed 5-9 years. Ventriculographic and other correlations. Circulation 1973;47:1154-63.

8 Cannon PJ, Stockley IH, Connell PA, Garner ST, Hampton JR. Prevalence of angina as assessed by a survey of nitrate prescriptions. Lancet 1988:979-81.

9 Sanmarco ME, Pontius S, Selvester RH. Abnormal blood pressure response and marked ischaemic ST-segment depression as predictors of severe coronary artery disease. Circulation 1980;61:572-8.

10 Bobbio M, Pollock BH, Cohen I, Diamond GA. Comparative accuracy of clinical tests for diagnosis and prognosis of coronary artery disease. Am $\mathcal{F}$ Cardiol 1988; 62:896-900.

11 The health of the nation. A consultative document for England and Wales. London: HMSO, 1991. 